\title{
Respiratory advice for the non-respiratory physician in the time of COVID-19
}

\author{
Authors: Jonathan Bennett, ${ }^{A}$ Mohammmed Munavvar, ${ }^{B}$ Paul Walker ${ }^{C}$ and Gerrard Phillips ${ }^{D}$
}

COVID-19, the disease caused by the SARS-CoV-2 betacoronavirus, has changed clinical practice in a matter of weeks. Among the physician specialties, respiratory physicians have been at the forefront of the response to this new challenge. Here we provide advice for non-respiratory physicians on the ward-based care of patients with this disease. This includes recommendations on hydration, thromboprophylaxis, nutritional support and on the importance of the early detection of deterioration, setting ceilings of care and use of anticipatory drugs where appropriate. We also discuss oxygen support modalities, proning, safe working practices and a new approach to multi-professional working. We include references to a number of important research studies.

KEYWORDS: Respiratory, COVID-19, coronavirus

DOI: $10.7861 /$ clinmed.2020-0188

\section{Introduction}

The novel Wuhan beta-coronavirus, SARS-CoV-2, which causes the disease COVID-19, has changed clinical practice in a matter of weeks. These are exceptional times, and clinicians are having to keep up with rapidly changing information, with new knowledge emerging daily.

We are now aware that the disease has different phases, with an initial flu-like illness during the first 5 days followed by the potential for COVID-19 pneumonia between days 5 to 10 . Once the symptoms of COVID-19 pneumonia appear, a patient's condition may deteriorate very quickly, with some patients moving from low to extremely high oxygen requirements and/or critical care within hours. Patients appear to experience less difficulty in breathing but are more hypoxaemic than usual. They tend to have increased respiratory rates and high tidal volumes and, in addition, the increased tidal volumes and high oxygen concentrations required to support them may propagate lung injury.

The median time for intubation and ventilation of a patient with COVID-19 is 10 days and the mortality rate for ventilated patients

Authors: ${ }^{A}$ chair, British Thoracic Society, London, UK; ${ }^{B}$ president, British Thoracic Society, London, UK; Ctreasurer, British Thoracic Society, London, UK; Dexecutive medical director, Federation of Royal Colleges of Physicians of the UK, London, UK may be up to $50 \%$, or even higher. Many of these patients require complex cardiovascular support, with some also requiring renal replacement therapy.

The nature of the viral pneumonia/acute respiratory distress syndrome (ARDS) that these patients experience is different from 'usual' ARDS and requires a different approach. There is currently uncertainty as to whether early or late intubation is better, with different UK intensive care units favouring different approaches. Hopefully, data on this will be available soon. However, planned intubation is advisable, and emergency intubation should be avoided if at all possible, because of the risk to healthcare workers. Early PEEP (positive end-expiratory pressure - see glossary, Box 1), high PEEP and spontaneous ventilation appear to be harmful. Proning is important. Prostacyclin and inhaled nitric oxide may have a role. Patients should be run euvolaemic rather than dry. By the time they come to critical care, they may have been pyrexial and hyperventilating for several days and so are often underfilled. There appears to be a higher rate of acute (or acute on chronic) kidney injury, requiring renal support. This may be due to a combination of being underfilled, injudicious use of diuretics, PEEP and renal circulation micro-thrombosis. Disseminated intravascular coagulation (DIC) and thrombosis within the pulmonary circulation are common, and the latter may or may not be embolic. High reintubation rates seem to occur, often due to airway swelling, so leak testing is important and patients should not be extubated too early.

Given these differences with COVID-19, the approach to patient management in the non-ITU setting also requires changing, with practice implications for the non-respiratory (and respiratory) physician, which we outline here. We are having to abandon, temporarily, many years of established, well-founded practice as being irrelevant for this new disease entity. With COVID-19 pneumonia, antibiotics have a limited role, the stethoscope adds little to the assessment process except in some patients with airways disease (asthma and COPD), and monitoring is done with oxygen saturation rather than blood gases (except in patients at risk of hypercapnic respiratory failure).

\section{Fundamentals of care: some specific points}

The fundamental treatment for COVID-19 pneumonia is supportive, comprising oxygen delivery, hydration and symptom control.

For both respiratory and non-respiratory physicians looking after these patients, it is clearly important to avoid

> dehydration: the adage 'run the urea in the normal range' where this is possible and appropriate is a useful guide 
> venous thrombosis: there is evidence that patients with COVID-19 are at increased risk, so attention to good quality thomboprophylaxis is important. A recent report of 1,026 patients from China determined that 407 (40\%) met the Padua Prediction Score for high venous thromboembolism risk. Both the British Thoracic Society ${ }^{2}$ and Thrombosis UK ${ }^{3}$ have produced useful guidance.

\section{The importance of early recognition of deterioration}

It is vitally important to be able to recognise deterioration in the patient's condition quickly and early, using the NEWS2 score, ${ }^{4}$ and to intervene swiftly and appropriately. Timing, at this point, can make all the difference to the patient's trajectory. If patients are requiring $>10-15 \mathrm{l} /$ min oxygen to maintain adequate oxygen saturation (some would set the threshold lower than this) then they should be started on continuous positive airway pressure (CPAP; see glossary, Box 1) and transferred to the critical care setting early if they are candidates for intubation. Note that the scoring for oxygen in the RCP's NEWS2 algorithm is binary (yes/no), meaning that a further significant change in oxygen requirement may not increase the score. Therefore it is important to remember that any significant increase in oxygen requirement in a COVID-19 suspected/proven patient should trigger an immediate escalation call to a senior decision maker.

\section{Ceilings of care}

We all know critical care is not a benign therapy and that survivors have the potential to suffer from ongoing problems. Patients need to be fitter than usual to have a reasonable chance of surviving a critical care stay due to COVID-19.

Given the manner in which patients can deteriorate rapidly we are now expected to make crucial decisions regarding ceilings of care at the start of the patient's hospital admission. Ideally, this should be done in concert with critical care colleagues, who are accumulating experience of which patients do well, and which do not, with critical care. Making early decisions may feel uncomfortable in some instances, but recommendations are in place regarding such assessment. There are a number of algorithms, and methodologies may vary between trusts, but a clinical frailty score, such as the Rockwood Frailty Score, ${ }^{5}$ is a core assessment tool. However, this should not be used in isolation. There is no substitute for clinical judgement in making appropriate escalation decisions.

If the outcome of the initial assessment is that the patient is not for escalation to the critical care setting, we are now being expected to prescribe anticipatory medicines at the time of the initial assessment. ${ }^{6}$

Our compassion and ability to communicate these difficult messages must be at the core of all that we do.

\section{Safe working practices}

The traditional structures of ward working have been modified as a highly infectious agent is already taking a significant toll on healthcare workers.

\section{SPACES}

One strategy, promoted by the British Thoracic Society, Royal College of Physicians and Royal College of Nursing, is SPACES, a standardised approach to the management of ward care that uses the mnemonic 'Sharing Patient Assessments Cuts Exposure for Staff' (Fig 1).? It is based on the principle of 'maximum patient

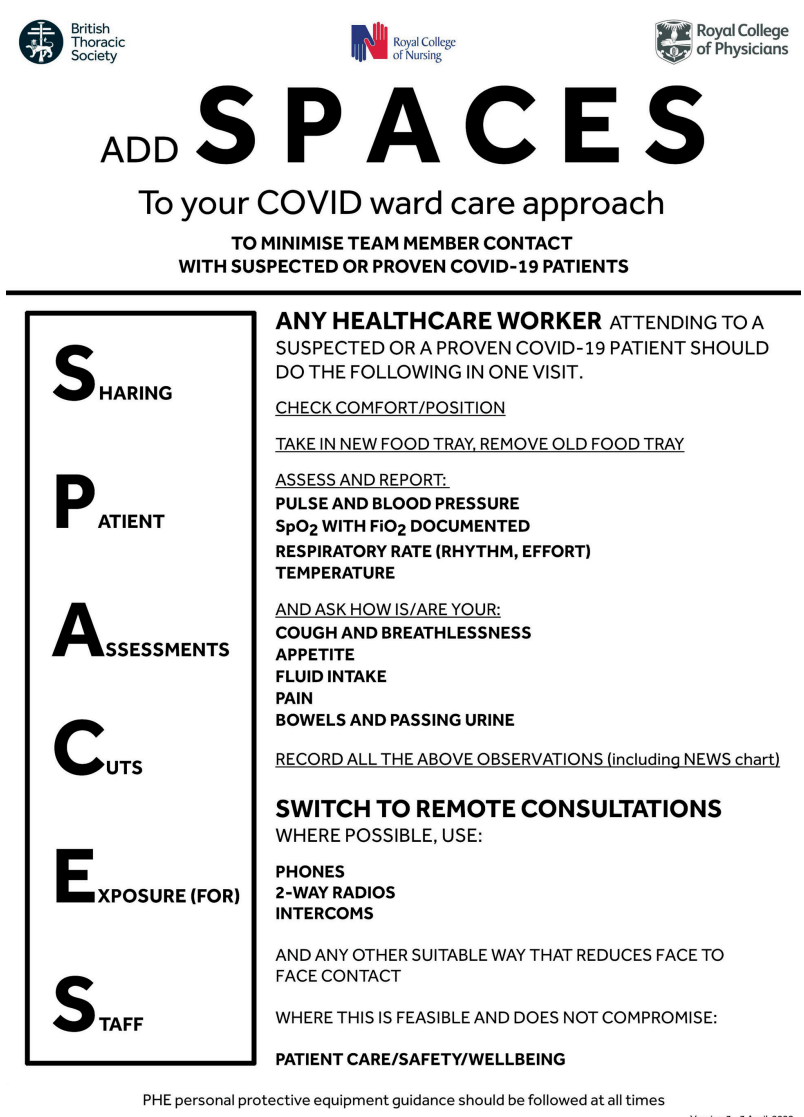

Fig 1. SPACES poster for ward display.

contact - minimum staff exposure'. Adopting SPACES can help to keep staff safe and to reduce the use of the personal protection equipment (PPE) required when caring for COVID-19 patients (although all healthcare workers should ensure they follow Public Health England guidance on PPE use at all times ${ }^{8}$ ).

SPACES emphasises the need for multi-professional working, maximising each staff-patient interaction and thereby reducing overall staff exposure to the virus. It reduces multiple entries into rooms for 'standard' procedures, observations and assessments. Any healthcare worker making patient contact can deliver all the most basic aspects of care, check position and comfort, perform symptom assessment and basic observations and document these in the patient's record.

In addition, to further reduce exposure risks, ward staff should switch to remote assessments and consultations wherever possible, using phones, intercoms, two-way radios and any other suitable method that reduces face-to-face contact - as long as this does not compromise the quality of care, patient safety and wellbeing.

\section{Cardiopulmonary resuscitation}

PPE with cardiopulmonary resuscitation (CPR) has been a source of much discussion and contention. Advice from national bodies, such as Public Health England and the Resuscitation Council (UK), differs. ${ }^{9}$ The Royal College of Physicians supports the Resuscitation Council (UK) advice. However, the reality is that a patient progressing to cardio-respiratory arrest due to progressive COVID-19 has an appalling in-hospital mortality rate, based on the evidence from Wuhan. ${ }^{10}$ 
This emphasises the importance of the decision-making strategy detailed previously regarding escalation planning early in the patient hospital journey.

\section{Oxygen practice}

\section{New saturation ranges}

We are using different acceptable normal ranges for oxygen. Prescribing targets for adults treated in NHS hospitals should be adjusted from the current 'standard' oxygen saturation range of $94-98 \%$ to $92-96 \%$ in the first instance for all patients. This should help conserve hospital oxygen supplies. In addition, evidence from clinical trials suggests that hyperoxia may be harmful and lower oxygen target ranges are safe. A lower target range of $90-94 \%$ may be considered if clinically appropriate by hospitals according to prevailing oxygen flow demands. ${ }^{11}$

\section{More use of CPAP}

Clinicians are having to learn quickly the full range of oxygen delivery systems. The number of patients attending with hypercapnic respiratory failure appears to be limited and high tidal volumes may be harmful, hence the use of CPAP rather than non-invasive ventilation (NIV; see glossary in Box 1) is advocated for the vast majority of patients, although both are categorised as aerosol-generating procedures (AGP). Coupled to this is the risk that oxygen supplies may be overwhelmed by demand. The earlier use of CPAP, and the redesign of non-invasive ventilators as CPAP delivery devices (where IPAP equals EPAP - see Box 1 - with entrained oxygen), reduces demand on hospital VIEs (vacuum insulated evaporators; see Box 1). Guidance on the role and use of non-invasive respiratory support in adult patients with confirmed or suspected COVID-19 has been produced by NHS England. ${ }^{12}$

Guidance for tailoring respiratory support of COVID-19 patients on medical wards according to prevailing hospital oxygen flow capacity and ventilator capacity has been produced by the British Thoracic Society, and includes scenarios in which conservation of the hospital oxygen supply is pressing. ${ }^{13}$ Liaison with hospital engineers can help clinicians to understand the limitations on oxygen delivery in their individual hospital.

CPAP is being regularly used as a ceiling of treatment, a trial to avoid intubation or to facilitate extubation. The CPAP set up must have a viral filter, appropriately placed, on the expiratory circuit and this needs to be changed regularly to reduce AGP and staff exposure and, for the same reason, non-vented masks must be used. The set-up is shown in guidance from NHS England ${ }^{12}$ and the British Thoracic Society. ${ }^{14}$ Note that the British Thoracic Society document mentions NIV which is, by and large, no longer recommended, unless machines are used in CPAP mode (to which they can be converted, see above). Note also that attaching the oxygen supply to the face mask itself, rather than to the ventilation circuit, may help conserve oxygen supplies.

The Association for Respiratory Technology and Physiology (ARTP) has recently produced additional guidance for CPAP set up and data supporting the use of CPAP, including its effects on $\mathrm{FiO}_{2}$ and hospital oxygen supplies. ${ }^{15}$

CPAP settings have been recommended to be at a pressure of $10 \mathrm{~cm} \mathrm{H}_{2} \mathrm{O}$ with $\mathrm{FiO}_{2}$ of $60 \%$; however, there is concern that this could propagate lung injury and hence lower settings may be better, at least initially, depending on how ill the patient is. This may also help patients to acclimatise to CPAP, and therefore to tolerate it better. Regular review of the patient with respect to response to CPAP and the fit and tolerability of the mask is important. The COVID-19 pneumonia can take several days to improve, as seen in ITU, and so some patients may be committed to several days of non-invasive pressure support oxygen delivery. We advocate cohorting of CPAP patients if possible.

\section{Which non-invasive respiratory support modality}

At present, in the UK, the recommended support modality if conventional oxygen is insufficient and invasive ventilation is not required is CPAP. ${ }^{12}$ Venturi masks are not recommended because they require a higher oxygen flow which, as mentioned previously, may compromise the oxygen supply of the hospital. CPAP with entrained oxygen achieves similar or better $\mathrm{FiO}_{2}$ at lower oxygen flow rates. However, several international guidelines recommend high-flow nasal cannula oxygen (HFNCO; see glossary, Box 1) as the preferred step up from conventional oxygen and NIV if HFNCO is not available. ${ }^{16,17}$ At least one of these guidelines recommends full PPE and the ITU environment for HFNCO. ${ }^{17}$ High quality evidence on the efficacy of these modalities in COVID-19 is lacking; therefore a number of organisations have set up the RECOVERY-RS study, a UK study which will compare standard oxygen care with CPAP and HFNCO in patients with an $\mathrm{FiO}_{2} \geq 0.4$ and $\mathrm{SpO}_{2} \leq 94 \%$ where there is a plan for escalation to ventilation if needed. ${ }^{18}$ Many centres have devised their own management aids. Figs 2 and 3 show two complementary flow charts developed by University Hospitals, Leicester.

\section{Proning}

We have also seen the introduction of non-ITU 'proning' of patients. For patients who need an $\mathrm{FiO}_{2} \geq 0.28$ and/or who require more than basic respiratory support to achieve $\mathrm{SpO}_{2}$ 92-96\% (88$92 \%$ if there is a risk of hypercapnic respiratory failure), and who have suspected/confirmed COVID-19, clinicians should consider the prone position if the patient is able to communicate and cooperate with this, is able to rotate onto their front, can adjust their position independently and has no anticipated airway issues.

The suggested regimen is as follows. ${ }^{19}$ Monitor oxygen saturations for 15 minutes: if the $\mathrm{SpO}_{2}$ is $92-96 \%$ and there is no obvious distress, continue the proning process, changing position every 2 hours according to the schedule below, aiming to achieve a prone time that is as long as possible. When not prone, aim for the patient to be sat at between 30-60 degrees upright. Monitor oxygen saturations after every position change and titrate down oxygen requirements as able.

The suggested position schedule, to be repeated as long as proning is indicated to continue, is:

$>30$ minutes to 2 hours lying fully prone (bed flat)

$>30$ minutes to 2 hours lying on right side (bed flat)

> 30 minutes to 2 hours sitting up (30-60 degrees) by adjusting the head of the bed

> 30 minutes to 2 hours lying on left side (bed flat).

\section{Patients with airways disease}

Thus far it does not appear that COVID-19 is associated with an increased risk of airways exacerbations in patients with asthma or COPD. Rather, it is predominantly an alveolar pneumonitis process. However, should a patient with airways disease have an 


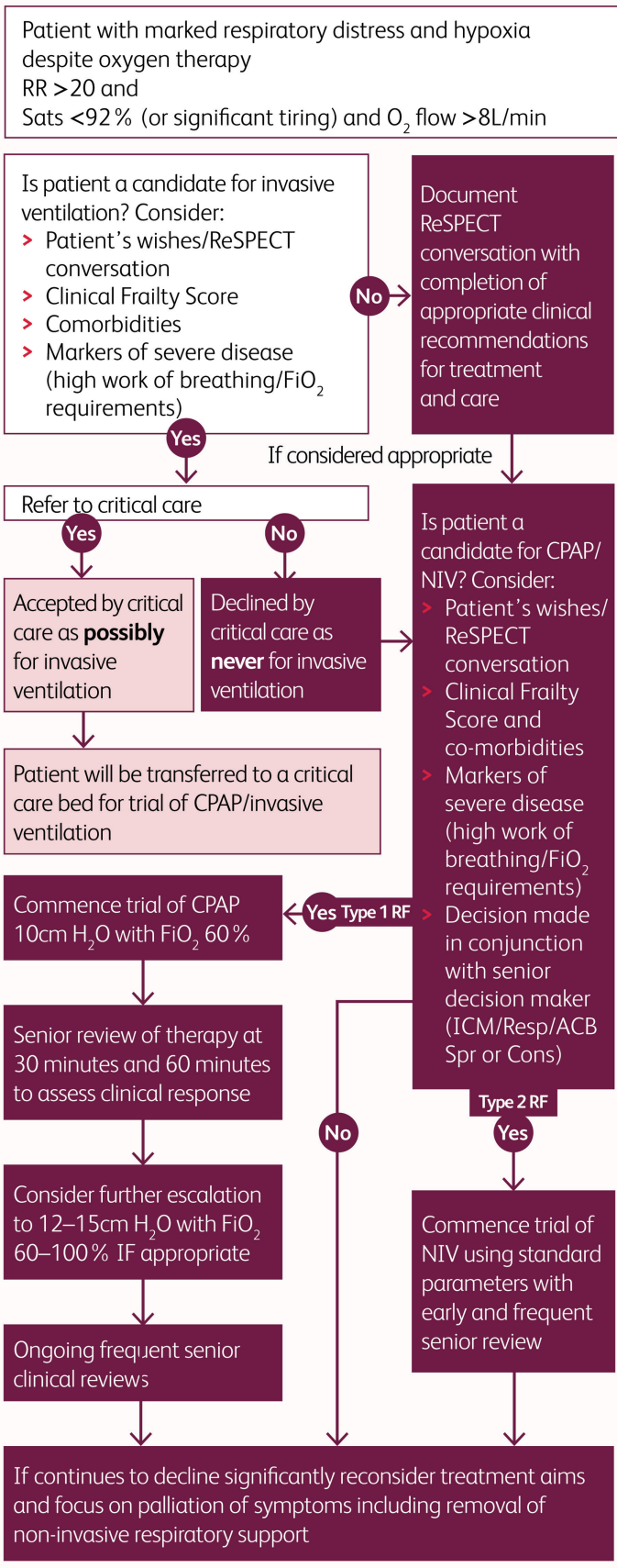

Ward $\square$ ICU

Fig 2. Example of a local management aid: escalation of respiratory support decision aid. Developed by S Knapp, L Walker, J Parker and M Steiner for University Hospitals, Leicester. CPAP = continuous positive airway pressure, NIV = non-invasive ventilation, $\mathrm{RF}=$ respiratory failure, $\mathrm{RR}=$ respiratory rate.

exacerbation they should be treated as per 'standard' therapy with steroids and bronchodilators. There has been much social media concern regarding nebulisers being a potential AGP source, but the limited evidence suggests that nebulisation is not a viral droplet generating procedure; instead, the droplets come from the machine (liquid bronchodilator drug particles) and not the patient.

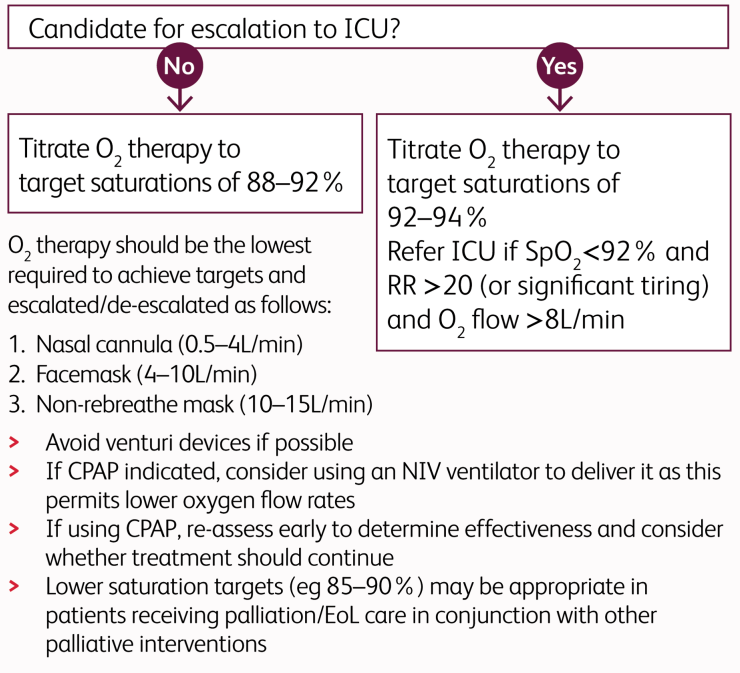

Fig 3. Example of a local management aid: use of oxygen therapy for adult inpatients during COVID-19 pandemic - clinical guidance to help ensure efficient usage of oxygen. Developed by S Knapp, L Walker, J Parker and M Steiner for University Hospitals, Leicester. CPAP = continuous positive airway pressure, EoL = end of life, NIV = non-invasive ventilation.

\section{Nutrition}

The British Association for Parenteral and Enteral Nutrition (BAPEN), in association with the British Thoracic Society and others, has produced customised advice on nutritional support for COVID-19 patients, with a particular focus for those on CPAP. ${ }^{20}$

\section{Research programmes}

Coupled to these new approaches to patient management, the pandemic has prompted the largest acute research programme ever undertaken in the UK. There are currently three main trials:

> PRINCIPLE: a platform trial of interventions against COVID-19 in older people

> RECOVERY: a trial to evaluate if existing or new drugs can help patients hospitalised with confirmed COVID-19

> REMAP-CAP: a platform trial for severely ill patients with COVID-19.

\section{Closing remarks}

We are in strange and stressful times, requiring extraordinary changes to our practice, some of which feel completely alien, adding to our sense of discomfort. We shall probably all make some mistakes along this journey and have feelings of being overwhelmed at some point, but we are continuing to learn rapidly and thereby hopefully will improve the frequency with which we are able to make the correct decisions, for both our patients and for our healthcare colleagues.

\section{Acknowledgements}

SPACES was developed in collaboration with a multidisciplinary respiratory team consisting of nurses, consultants and other healthcare professionals based at Glenfield Hospital, University Hospitals of Leicester NHS Trust. The team's original idea was further refined into the final SPACES approach by the British 


\section{Box 1: Glossary of terms}

Continuous positive airway pressure (CPAP) - the additional pressure applied to the lungs (alveoli) above atmospheric pressure throughout the respiratory cycle. This applies a single, fixed pressure throughout inspiration and expiration, which does not vary, via a tightly fitted nasal or whole face mask to the spontaneously breathing, conscious patient without the need for intubation and sedation. This is not a form of ventilation High-flow nasal cannula oxygen therapy (HFNCO) - this is an oxygen supply system capable of delivering up to $100 \%$ humidified and heated oxygen at a flow rate of up to 60 liters per minute via nasal cannulae

Non-invasive ventilation (NIV) - this delivers mechanically supported breaths via a tightly fitted nasal or whole face mask to the spontaneously breathing, conscious patient without the need for intubation and sedation. The patient usually triggers the inspiratory cycle. Expiration is passive, by elastic recoil. The pressure delivered varies during the respiratory cycle, being higher in inspiration than expiration. This is a form of ventilation

IPAP - Inspiratory positive airway pressure, the inspiratory pressure set on the non- invasive ventilator

EPAP - Expiratory positive airway pressure, the expiratory pressure set on the non-invasive ventilator

Positive end expiratory pressure (PEEP) - the additional pressure applied to the lungs (alveoli) above atmospheric pressure at the end of expiration

Vacuum-insulated evaporator (VIE) - the common method for hospital storage of liquid oxygen. The inner shell is of stainless steel and is separated from the outer carbon shell by a vacuum. The contents are at a pressure of 700-1000 KPa and a temperature of $-160 \mathrm{C}$ to $-180 \mathrm{C}$ (the critical temperature for oxygen is $-118 \mathrm{C}$ )

Thoracic Society through the involvement of a small expert group of Respiratory Physicians, and with feedback and endorsement from the Royal College of Physicians and the Royal College of Nursing.

\section{References}

1 Wang T, Chen R, Liu C et al. Attention should be paid to venous thromboembolism prophylaxis in the management of COVID19. Lancet Haematol 2020, in press (DOI: 10.1016/S23523026(20)30109-5.

2 British Thoracic Society. BTS guidance on venous thromboembolic disease in patients with COVID-19. BTS, 2020. Available from www. brit-thoracic.org.uk/about-us/covid-19-information-for-the-respiratory-community/.

3 Hunt B, Retter A and McClintock C. Practical guidance for the prevention of thrombosis and management of coagulopathy and disseminated intravascular coagulation of patients infected with COVID-19. Thrombosis UK, 2020. Available from: https://thrombosisuk.org/covid-19-thrombosis.php.

4 Royal College of Physicians. National Early Warning Score (NEWS) 2: Standardising the assessment of acute-illness severity in the NHS. RCP, 2017. Available from: www.rcplondon.ac.uk/projects/ outputs/national-early-warning-score-news-2.

5 Rockwood K, Song X, Macknight C et al. A global clinical measure of fitness and frailty in elderly people. CMAJ 2005;173:489-95.

6 National Institute for Health and Care Excellence. COVID-19 rapid guideline: critical care in adults. NICE guideline [NG159]. NICE, 2020. Available from: https://www.nice.org.uk/guidance/ng159.

7 British Thoracic Society. Add SPACES to your COVID ward care approach. BTS, 2020. Available from www.brit-thoracic.org.uk/ about-us/covid-19-information-for-the-respiratory-community/.

8 Public Health England. COVID-19 personal protective equipment (PPE). PHE, 2020. Available from: www.gov.uk/government/publications/wuhan-novel-coronavirus-infection-prevention-and-control/ covid-19-personal-protective-equipment-ppe [Accessed 21 April 2020].

9 Wyllie J.RCUK Statement on PHE PPE guidance. Resusitation Council UK, 2020. Available from: www.resus.org.uk/media/ statements/resuscitation-council-uk-statements-on-covid-19-coronavirus-cpr-and-resuscitation/statement-on-phe-ppe-guidance/\# [Accessed 23 April 2020].

10 Shao F, Xu S, Ma X. In-hospital cardiac arrest outcomes among patients with COVID-19 pneumonia in Wuhan, China. Resuscitation 2020;151:18-23.

11 NHS England. Clinical guide for the optimal use of oxygen therapy during the coronavirus pandemic. NHS England, 2020. Available from: www.england.nhs.uk/coronavirus/wp-content/uploads/ sites/52/2020/04/C0256-specialty-guide-oxygen-therapy-andcoronavirus-9-april-2020.pdf.

12 NHS England. Guidance for the role and use of non-invasive respiratory support in adult patients with COVID19 (confirmed or suspected). NHS England, 2020. Available from: www.england. nhs.uk/coronavirus/wp-content/uploads/sites/52/2020/03/specialty-guide-NIV-respiratory-support-and-coronavirus-v3.pdf.

13 British Thoracic Society. Respiratory support of patients on medical wards. BTS, 2020. Available from: www.brit-thoracic.org.uk/document-library/quality-improvement/covid-19/bts-guidance-respiratory-support-of-patients-on-medical-wards/.

14 British Thoracic Society. Use of acute NIV in patients hospitalised with suspected or confirmed COVID-19 infection. Available from: www.brit-thoracic.org.uk/media/455095/advice-on-acute-niv-technical-aspects-final-16-march-2020.pdf.

15 Association for Respiratory Technology and Physiology. Data on CPAP and entrained oxygen. ARTP, 2020. Available from www.artp. org.uk/write/MediaUploads/Standards/COVID19/Data_on_CPAP_ and_entrained_oxygen.pdf.

16 Alhazzani W, Møller MH, Arabi YM et al. Surviving sepsis campaign: guidelines on the management of critically ill adults with coronavirus disease 2019 (COVID-19). Intensive Care Med 2020, in press (DOI:10.1007/s00134-020-06022-5).

17 Australian and New Zealand Intensive Care Society. COVID-19 guidelines, version 2. ANZICS, 2020. Available from: www.anzics. com.au/wp-content/uploads/2020/04/ANZI_3367_Guidelines_ V2.pdf.

18 RECOVERY-RS Respiratory Support: respiratory strategies in COVID19; CPAP, high-flow, and standard care. Warwick University, 2020. Available from: https://warwick.ac.uk/fac/sci/med/research/ctu/ trials/recovery-rs/.

19 Intensive Care Society. ICS guidance for prone positioning of the conscious COVID patient 2020. ICS, 2020. Available from: https:// emcrit.org/wp-content/uploads/2020/04/2020-04-12-Guidancefor-conscious-proning.pdf.

20 British Association for Parenteral and Enteral Nutrition. Route of nutrition support in patients requiring NIV \& CPAP during the COVID-19 response. BAPEN, 2020. Available at: www.bapen.org.uk/ pdfs/covid-19/nutrition-in-niv-21-04-20.pdf.

Address for correspondence: Dr Gerrard Phillips, Department of Respiratory Medicine, Dorset County Hospital, Williams Ave, Dorchester DT1 2JY, UK.

Email: gerrard.phillips@dchft.nhs.uk 